\title{
Multi-analyte electrochemical biosensors on flexible foils
}

G. Zambrano ${ }^{1}$, V. Di Palma ${ }^{2}$, M. F. Bevilacqua ${ }^{2}$, A. di Matteo ${ }^{2}$, V. Pavone ${ }^{1}$, O. Maglio ${ }^{1,3}$, A. Lombardi ${ }^{1}$ 'Dept. of Chemical Science, University of Naples "Federico II", via Cintia, 80126 Napoli, Italy,

${ }^{2}$ STMicroelectronics, Via Remo de Feo 1, 80022 Arzano (NA), Italy

${ }^{3}$ IBB, CNR, via Mezzocannone 16, 80134 Napoli, Italy

gerardo.zambrano@unina.it

\begin{abstract}
Enzyme-based electrodes represent an important class of biosensors that allow to electrochemically measure the products derived from a target analyte transformation. Enzymatic electrochemical biosensors, capable to detect glucose and lactate, find application in monitoring diseases, such as diabetes. This work is focused onto the development of an amperometric multy-analyte (glucose and lactate) biosensor based on glucose and lactate oxidase immobilization, loaded into photo-patternable hydrogels. The photo-definable hydrogels act as excellent biocompatible matrices providing near-physiological conditions that minimize protein denaturation. This methodology also allows biosensor miniaturization for a continuous non-invasive glucose/lactate monitoring.
\end{abstract}

Key words: multi-analyte amperometric sensors, hydrogels, glucose, lactose, diabetes.

\section{Introduction}

The traditional strips for plasmatic glucose and lactate monitoring involve sticking finger to gain access to analytes in a onetime measurement. This episodic monitoring does not provide information about fast fluctuations in two consecutive level measurements and originates mismanagements in diabetes followup.

Nowadays, innovative approaches are oriented to achieve a continuous glucose and lactate monitoring. Dynamic blood-sugars monitoring may help not only in decreasing hypo- and hyperglycemia episodes, but also to avoid life-threatening diabetic complications, such as retinopathy, kidney damage, heart diseases, stroke, neuropathy and birth defects. ${ }^{2}$ In this field, amperometric biosensor based on natural enzymes may avoid complications given by wide fluctuations.

To this aim, photo-definable hydrogels are proposed as enzyme matrices, that ensure nearly physiological conditions, minimizing protein denaturation. Moreover, the three-dimensional geometry of hydrogels enables them to contain a large quantity of the sensing reagent, in a confined space, thanks to the lithographic approach, thereby increasing their signal-to-noise ratio and sensitivity.

In this work we present a second generation biosensors comprising a 3-electrode configuration array on flexible foils casted through a lithographic approach.

\section{Results and discussion}

The biosensor set up and the sensing procedure are summarized in Fig. 1.

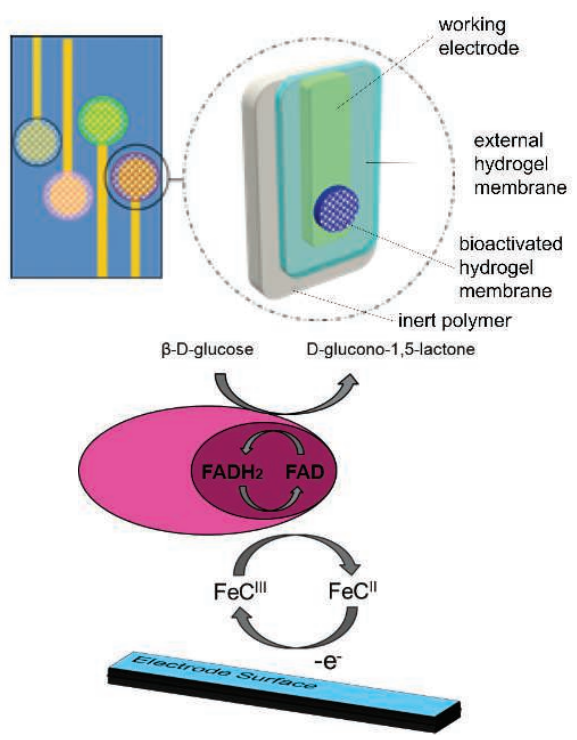

Fig. 1 Biosensor set up (up) and sensing pathway (down).

Vinylferrocene, used as redox mediator, shuttles the electrons from the enzyme/analyte reaction (Glucose Oxidase from Aspergillus Niger/D-Glucose or Lactate Oxidase from Pediococcus/Lactic Acid) through the hydrogel to the counter electrode. The hydrogel is constituted by a mixture of PolyEthylene GlycolDiAcrylate (PEG-DA) and 2-hydroxy-2methylpropriophenone as photoinitiator. The hydrogel with enzyme and mediator was selectively deposited on $\mathrm{Au}$ electrodes, and the surface was modified with an insulating organo-silane layer to ensure good adhesion between the hydrogel membrane and the inert polymer, avoiding water infiltration. 
The electrochemical features of the device were investigated by performing cyclic voltammetry (Fig. 2), with $\mathrm{K}_{3}\left[\mathrm{Fe}(\mathrm{CN})_{6}\right]$ as the diffusible redox species.

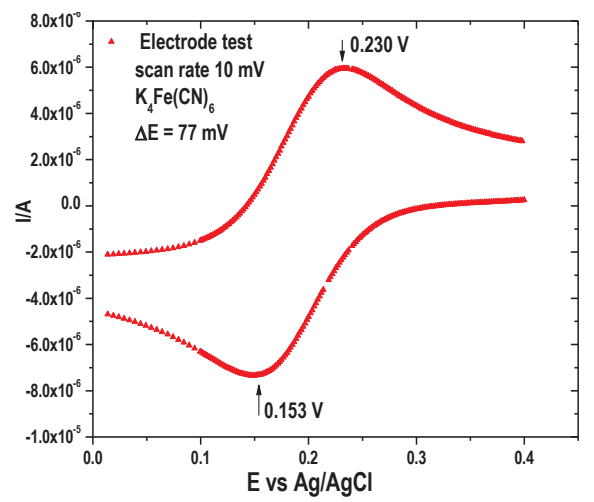

Fig. 2 Electron transfer test

Furthermore, cyclic voltammograms were acquired at different scan rates (Fig. 3) to evaluate vinylferrocene activity.

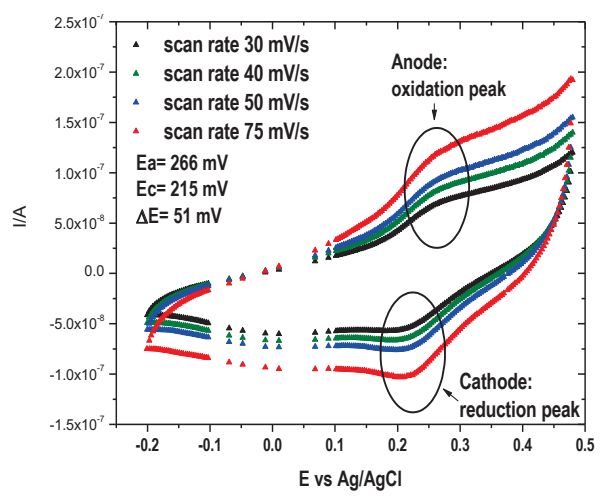

Fig. 3 Evaluation of biosensor behavior

Both tests were consistent with a good electrode response. In particular, they confirmed a fast and reversible vinylferrocene redox behavior in the matrix.

Amperometric response for glucose and lactate were also evaluated. A constant potential was applied to the electrochemical cell and the current response was monitored. Fig. 4 and Fig 5 show the response upon addition of $2 \mathrm{mM}$ and $1 \mathrm{mM}$ aliquots of glucose and lactate, respectively.

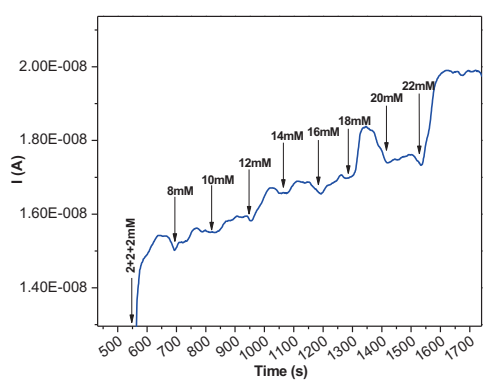

Fig. 4 Amperometric response with sequential addition of glucose

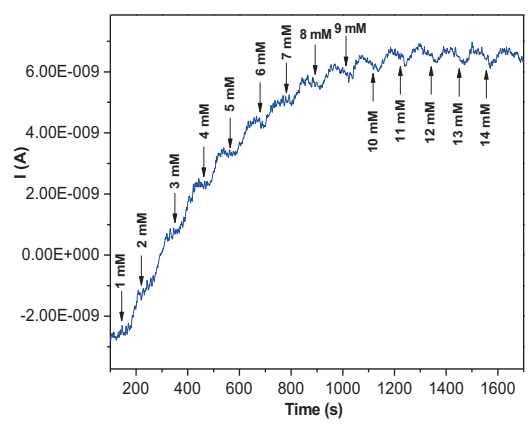

Fig. 5 Amperometric response with sequential additions of lactate

Both substrates were efficiently detected. In particular, glucose gave a linear response in the 8-22 $\mathrm{mM}$ range, while lactate in the 1-9 $\mathrm{mM}$ range. Sensitivity, calculated for both glucose and lactate, was $1 \mu \mathrm{A} \mathrm{cm}{ }^{-1} \mathrm{mM}^{-1}$.

These results confirm that the proposed device is promising for the detection of biomolecules in biological fluids.

Future activity will be aimed to increase sensitivity and linear range of detection.

\section{References}

[1] M. Hatada, N. Loew, Y. Inose-Takahashi, J. Okuda-Shimazaki, W. Tsugawa, A. Mulchandani, K. Sode, Development of a glucose sensor employing quick and easy modification method with mediator for altering electron acceptor preference, Bioelectrochemistry 121, 185-190 (2018); doi: 10.1016/j.bioelechem.2018.02.001

[2] S. M. Grundy, J. I. Cleeman, S. R. Daniels, K. A. Donato, R. H. Eckel, B. A. Franklin, D. J. Gordon, R. M. Krauss, P. J. Savage, S. C. Smith, J. A. Spertus, F. Costa, Diagnosis and Management of the Metabolic Syndrome, Circulation 112, 17, 2735-2752 (2005);doi: 10.1161/CIRCULATIONAHA. 105.169404

[3] J. Yan, V. A. Pedrosa, A. L. Simonian and A. Revzin, Immobilizing Enzymes onto Electrode Arrays by Hydrogel Photolithography to Fabricate Multi-Analyte Electrochemical Biosensors, Appl. Mat. \& Int. 2, 748-755 (2010); doi: 10.1021/am9007819

[4] N. Thomas, I. Lahdesmaki and B.A. Parviz, A contact lens with an integrated lactate sensor, Sens. and Act. B: Chemical, 162, 128-134 (2012); doi: 10.1016/ j.snb.2011.12.049 